\title{
MODELAGEM E SIMULAÇÃO DE MICROMISTURA EM CRISTALIZADOR COAXIAL UTILIZANDO OPENFOAM
}

\author{
C. ROMBALDI, L. F. I. FARIAS e C. A. DA ROSA \\ ${ }^{1}$ Universidade Federal do Rio Grande, Escola de Química e Alimentos \\ E-mail para contato: darosacezar@gmail.com
}

\begin{abstract}
RESUMO - A cristalização, em muitos processos, é a forma mais comum de produzir compostos químicos de alto valor agregado. Na indústria farmacêutica, o método de cristalização mais utilizado consiste na cristalização antissolvente, que tem a vantagem de induzir a cristalização de compostos que são facilmente degradados com o aumento da temperatura, sem necessitar de uma variação brusca da mesma. A fluidodinâmica computacional (CFD) é uma excelente ferramenta para simular, analisar e projetar sistemas de escoamentos complexos, como em fenômenos de micromistura, que ocorrem em cristalizadores. Sendo assim, este trabalho teve como objetivo a modelagem e simulação da micromistura em cristalizadores antissolvente de geometria coaxial, utilizando o método multi-escala, que acopla as equações dinâmicas do modelo RANS (Reynolds-Averaged Navier-Stokes) com um modelo de densidade de probabilidade (PDF) multiambiente. Além disso, avaliar a influência de diferentes condições operacionais na supersaturação relativa da solução. Para tanto, foram avaliadas três razões mássicas distintas, para proporção de antissolvente/solução de 1, 1,5 e 0,5, com base no modelo implementado no software livre OpenFOAM. As análises qualitativas e quantitativas mostraram que as razões de 1 e 1,5 apresentaram melhor desempenho tanto para macro quanto para micro mistura.
\end{abstract}

\section{INTRODUÇÃO}

A cristalização é uma técnica largamente utilizada em processos de separação sólidoliquido, no qual a formação dos cristais se dá através da supersaturação. Esta operação é baseada nos mecanismos de transferência de massa e quantidade de movimento. A formação dos cristais ocorre através da supersaturação na mistura líquida, ou seja, a existência de uma concentração de soluto na solução superior à concentração de saturação (limite de solubilidade), juntamente com a agitação desta mistura que provoca o movimento entre elas. A supersaturação gerada durante o processo, bem como o perfil de escoamento da mistura no cristalizador influencia diretamente nas características dos cristais, como tamanho, morfologia e pureza.

Existem diferentes métodos para atingir a supersaturação em um cristalizador através de processos físicos e químicos. O processo químico de cristalização antissolvente, consiste na adição de um segundo solvente (antissolvente) que reduz a solubilidade do soluto, também chamado drowning-out. Esta técnica de cristalização é amplamente utilizada na indústria farmacêutica devido a vantagem de induzir a cristalização de compostos que são facilmente degradados com o aumento da temperatura, sem necessitar de uma variação brusca da mesma. 
De acordo com estudos de Kaneco et al. (2002), a cristalização, incluindo a distribuição do tamanho de cristal (CSD), está diretamente relacionada às condições de funcionamento, tais como a taxa de adição e agitação, o modo de adição, a composição do solvente e o tamanho do cristalizador. Todavia, inúmeras combinações de condições operacionais podem ser investigadas, o que demanda demasiado tempo e investimento econômico para realização de experimentos. Sendo assim, a técnica CFD pode oferecer um conhecimento mais aprofundado dos fenômenos de micromistura envolvidos em cristalizadores antissolvente.

Alguns esforços já foram realizados no sentido de modelar e simular o fenômeno de micromistura em cristalizadores (Marchisio et al., 2001 a, b; Woo et al., 2009; Pirkle et al., 2015). No entanto, nos trabalhos destes autores a simulações foram conduzidas em geometrias bidimensionais, considerando as propriedades da mistura médias e constantes. Sendo assim, novos esforços são necessários para o aprimoramento dos modelos existentes e acoplamento destes com a técnica CFD.

Dentre os softwares, comerciais e gratuitos, disponíveis para o uso da técnica CFD, o pacote OpenFOAM, o qual é gratuito, surge como uma opção viável devido a sua simplicidade no uso como uma ferramenta CFD, e por possibilitar ao usuário a alteração do código fonte.

Desta foram, o objetivo do presente trabalho foi acoplar as equações da fluidodinâmica computacional com um modelo de micromistura multiambiente, utilizando o OpenFOAM, para simular a micromistura em cristalizadores coaxiais em 3D. Além disso, avaliar a influência de diferentes condições operacionais na micrimistura e na supersaturação da solução (soluto + solvente).

\section{MODELAGEM MATEMÁTICA}

A macromistura foi modelada através do modelo dinâmico RANS e o modelo de turbulência k-E padrão. As equações utilizadas para solução deste modelo são apresentadas abaixo.

Assim como nos trabalhos de Marchisio et al. (2001 a, b), Woo et al. (2009) e Pirkle Jr et al. (20015), os efeitos da micromistura foram considerados através do modelo PDF (Probability Distribution Function) multiambiente proposto por Fox (2003). Neste modelo, cada célula computacional é dividida em $N_{e}$ diferentes modos de probabilidade, chamados de ambientes, os quais correspondem a discretização da composição presumida em um número finito de funções delta $(\delta)$.

$$
f_{\phi}(\Psi ; \mathbf{x}, t)=\sum_{n=1}^{N_{e}} p_{n}(\mathbf{x}, t) \prod_{\alpha=1}^{N_{s}} \delta\left[\psi_{\alpha}-\left\langle\phi_{\alpha}\right\rangle_{n}(\mathbf{x}, t)\right]
$$

onde $f_{\phi}$ é a PDF agrupada de todas as variáveis escalares, $\mathrm{N}_{\mathrm{s}}$ é o número total de escalares (espécies), $P_{\mathrm{n}}$ é a probabilidade do modo n ou fração volumétrica ocupada pelo ambiente n, e $\left\langle\phi_{\alpha}\right\rangle_{n}$ é a composição média de $\alpha$ correspondente ao ambiente $\mathrm{n}$. A concentração ponderada na fração volumétrica do ambiente é dada por: 
$\langle\mathbf{s}\rangle_{n} \equiv p_{n}\langle\phi\rangle_{n}$

O transporte das probabilidades dos diferentes ambientes e de massa das espécies, em escoamentos não homogêneos, foi modelada por:

$$
\begin{aligned}
& \frac{\partial \mathbf{p}}{\partial t}+\sum_{i}\left[\left\langle v_{i}\right\rangle \frac{\partial \mathbf{p}}{\partial x_{i}}-\frac{\partial}{\partial x_{i}}\left(D_{t} \frac{\partial \mathbf{p}}{\partial x_{i}}\right)\right]=\mathbf{G}(\mathbf{p})+\mathbf{G}_{\mathbf{s}}(\mathbf{p}) \\
& \frac{\partial\langle\mathbf{s}\rangle_{n}}{\partial t}+\sum_{i}\left[\left\langle v_{i}\right\rangle \frac{\partial\langle\mathbf{s}\rangle_{n}}{\partial x_{i}}-\frac{\partial}{\partial x_{i}}\left(D_{t} \frac{\partial\langle\mathbf{s}\rangle_{n}}{\partial x_{i}}\right)\right]=\mathbf{M}^{n}\left(\mathbf{p},\langle\mathbf{s}\rangle_{1}, \vec{\nu},\langle\mathbf{s}\rangle_{N_{e}}\right)+\mathbf{M}_{\mathbf{s}}^{n}\left(\mathbf{p},\langle\mathbf{s}\rangle_{1}, \overrightarrow{,},\langle\mathbf{s}\rangle_{N_{e}}\right)+p_{n} \mathbf{S}\left(\langle\phi\rangle_{n}\right)
\end{aligned}
$$

onde $\mathbf{G}$ e $\mathbf{M}^{\mathrm{n}}$ são as taxas de variação de $\boldsymbol{P}=\left[P_{1}, P_{2}, \ldots, P_{N}\right]$ e $\langle S\rangle_{n}$ devido a micromistura, respectivamente; $\mathbf{G}_{\mathbf{s}}$ e $\boldsymbol{M}_{s}^{n}$ são termos adicionais para eliminar a dissipação espúria na equação de transporte da variância da fração de mistura (veja Fox (2003) para mais detalhes); e S é o termo fonte de geração ou consumo de massa.

Neste trabalho, um modelo de três ambientes foi utilizado para modelar os efeitos de micromistura. Assim, a solução (soluto + solvente) forma o Ambiente 1, o antissolvente representa o Ambiente 2 e a mistura o Ambiente 3. Segundo Marchisio et al. (2001 a, b), o uso de três ambientes é suficiente ara capturar os efeitos de micromistura e escoamentos não desenvolvidos com precisão satisfatória.

\section{ESTUDO DE CASO}

O modelo implementado no OpenFOAM foi aplicado na simulação de um cristalizador de geometria coaxial, utilizando a técnica de supersaturação antissolvente, em uma solução de lovastatina (soluto) e metanol (solvente), usando como antissolvente a água. Os dados cinéticos e de solubilidade da lovastantina foram baseados na literatura (Pirkle et al., 2015)

Foram consideradas três razões mássicas de alimentação (razão entre as vazões mássicas de água e solução) iguais a 0,5,1 e 1,5 para fins de comparação do desempenho do cristalizador. A vazão mássica total de $1,0 \mathrm{~kg} / \mathrm{s}$ foi mantida constante nas três condições de operação.

Um domínio 3D com simetria no plano $\left.\mathrm{YZ}\right|_{\mathrm{x}=0}$ foi utilizado para simular o cristalizador coaxial, conforme apresentado na Figura 1

Figura 1 - Domínio computacional.

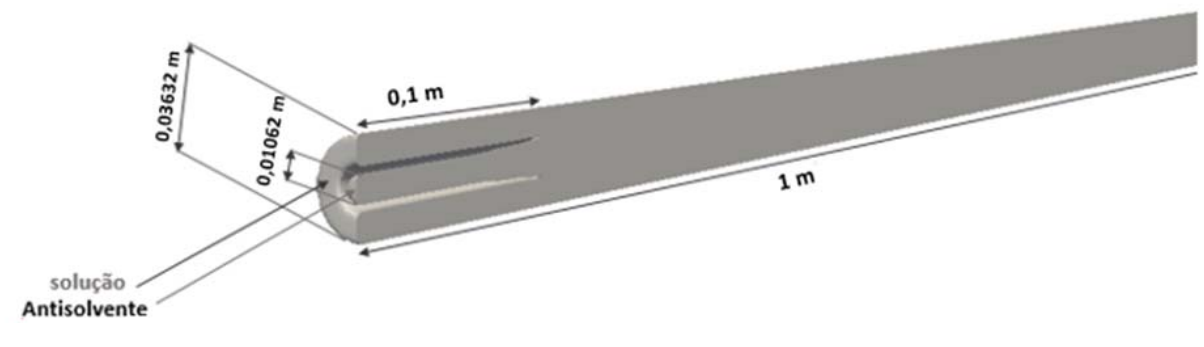




\section{SOLUÇÃO NUMÉRICA}

As equações do modelo foram implementadas no software OpenFOAM utilizando a linguagem orientada-objeto $\mathrm{C}++$. Foram criadas novas classes para o cálculo das propriedades da mistura, bem como para a solução das equações do modelo de micromistura considerando as propriedades da mistura variáveis.

A malha computacional 3D (Figura 2) foi gerada utilizando as ferramentas blockMesh e snappyHexMesh disponíveis no OpenFOAM. Foi gerada uma malha com 324 mil célula com predominância de células hexaédricas, com valores médio e máximo de não-ortogonalidade de 16 e 52, respectivamente.

Figura 2 - Malha computacional.

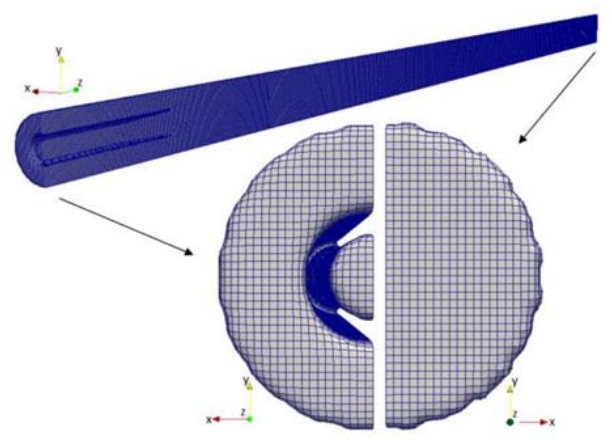

As simulações foram realizadas em regime transiente até que o regime permanente fosse alcançado. Os métodos de discretização implementados para ambos os termos, convectivos e difusivos, foram unbounded linear de segunda ordem. O acoplamento pressão velocidade foi realizado utilizando o algoritmo PIMPLE.

\section{RESULTADOS E DISCUSSÃO}

A Figura 3, apresenta a fração volumétrica do solvente (metanol) no plano de simetria YZ para três condições de operação propostas.

Figura 3 - Fração volumétrica de solvente no plano de simetria YZ para as diferentes razões de alimentação: (A) 0,5; (B) 1,0; e (C) 1,5.

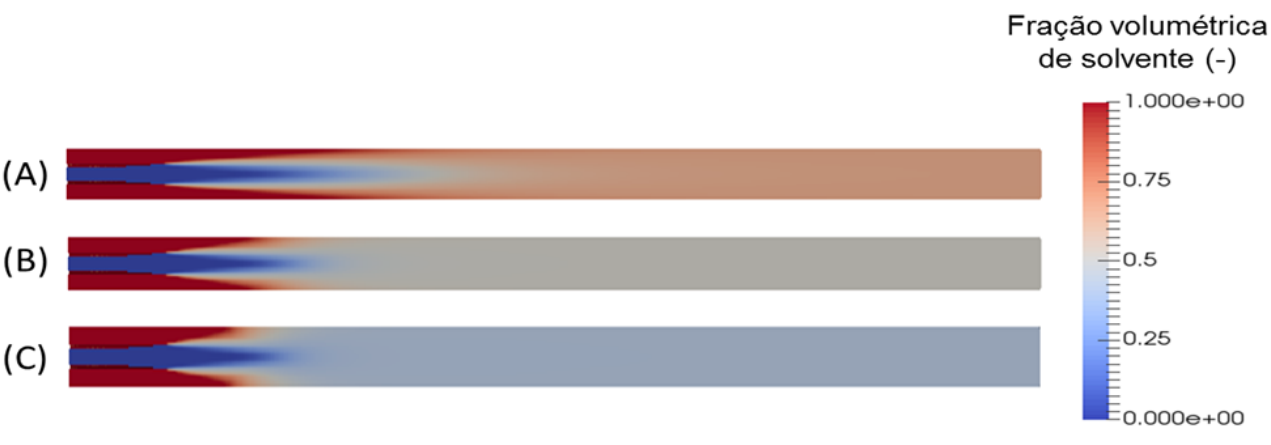


Como pode ser observado na Figura 3, para uma menor razão mássica $(0,5)$ de antissolvente/solução, tem-se uma alta concentração de metanol, enquanto que aumentando esta razão para 1,5, tem-se uma menor concentração de solvente. Levando em consideração o comportamento qualitativo da fração de solvente apresentado na Figura 3, pode-se observar que as condições (B) e (C) demonstram melhor macromistura, podendo ser observado pela ocorrência da mistura em um menor tempo de contato, enquanto que para a condição (A), necessita-se de um maior tempo de residência para que a mesma ocorra.

Este comportamento também pode ser observado na micromistura, conforme apresentado na Figura 4, a qual apresenta a média ponderada na massa da fração volumétrica do ambiente 3 (P3) em função da coordenada axial. A partir da Figura 4, é possível observar que o aumento da razão de alimentação antissolvente/solvente faz com que o desempenho do cristalizador com relação à micromistura seja melhor, alcançando uma fração de mistura elevada em menores comprimentos do cristalizador. Este comportamento está relacionado com a maior turbulência gerada pelo aumento da vazão mássica de antissolvente, a qual está diretamente relacionada com a transferência de massa na microescala.

Figura 4 - Fração volumétrica da mistura em função da coordenada axial.

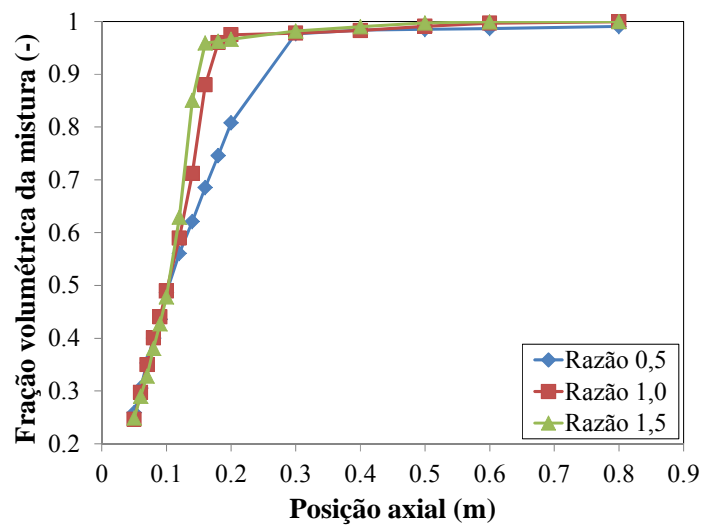

A melhor micromistura observada para as maiores razões de alimentação, aliada a maior fração de antissolvente no cristalizador, fazem com que a supersaturação relativa (força motriz da cristalização) seja maior nestas condições, conforme apresentado na Figura 5.

Figura 5 - Supersaturação relativa em função da coordenada axial.

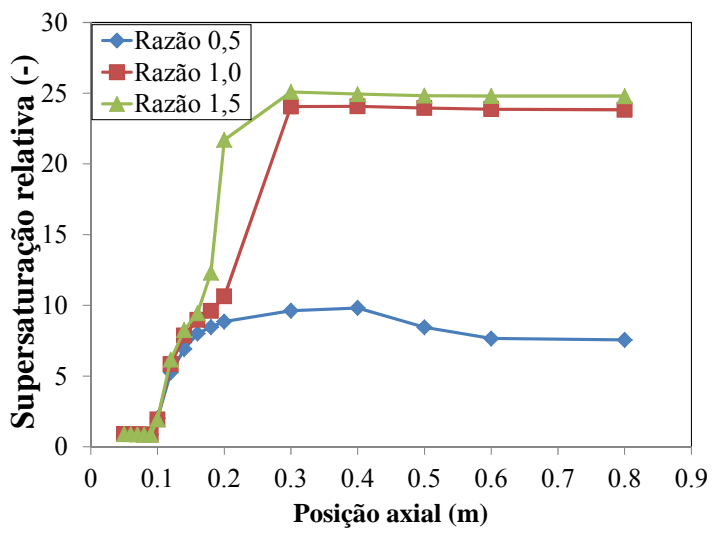




\section{CONCLUSÃO}

Com base nos resultados apresentados, pode-se concluir que foi possível implementar o modelo multi-escala e que este foi eficiente na simulação de diferentes condições operacionais. O software compilado com base no OpenFOAM mostrou-se estável em todas as simulações.

Maiores razão de alimentação entre antissolvente/solvente produziram uma melhor macro e micromistura, o que está relacionado com a maior turbulência gerada quando maiores quantidades de antissolvente são alimentadas ao cristalizador.

A melhor micromistura, aliada a maior quantidade de antissolvente, presentes na razões de alimentação de 1,0 e 1,5, proporcionaram maiores valores de supersaturação relativa, a qual é a força motriz da cristalização.

\section{REFERÊNCIAS}

Fox, R.O. "Computational Models for Turbulent Reacting Flows". Cambridge University Press, Cambridge, U.K, 2003.

KANEKO, S., YAMAGAMI, Y., TOCHIHARA, H., HIRASAWA, I. J. Effect of supersaturation on crystal size and number of crystals produced in antisolvent crystallization. Chem. Eng. Jpn. v. 35, p. 1219-1223, 2002.

MARCHISIO, D. L.; BARRESI, A. A.; FOX, R. O. Simulation of turbulent precipitation in a semi-batch Taylor-Couette reactor using CFD. AIChE J. v.47, p. 664-676, 2001.

MARCHISIO, D. L.; FOX, R. O; BARRESI, A. A.; BALDI, G. On the Comparison between Presumed and Full PDF Methods for Turbulent Precipitation. Ind. Eng. Chem. Res. v. 40, p. 5132-5139, 2001.

PIRKLE Jr., C.; FOGUTH, L. C.; BRENEK, S. J.; GIRARD, K.; BRAATZ, R. D. Computational fluid dynamics modeling of mixing effects for crystallization in coaxial nozzles. Chem. Eng. and Proc.: Proc. Int., v. 97, p. 213-232, 2015.

WOO, X. Y.; TAN, R. B. H.; BRAATZ, R. D. Modeling and computational fluid dynamicspopulation balance equation-micromixing simulation of impinging jet crystallizers. Cryst. Growth Des. v. 9, p. 154-164, 2009. 